\title{
Rôle des particules sur le comportement et la spéciation de métaux traces: exemple du cadmium
}

\author{
The role of particles in the behavior and speciation of trace metals: \\ the cadmium example
}

\author{
J-L. GONZALEZ, B. THOUVENIN, C. DANGE
}

IFREMER, Département «Biogéochimie \& Ecotoxicologie » Z. P. de Brégaillon B.P. 33083507 La Seyne sur Mer e-mail : gonzalez@ifremer.fr

\begin{abstract}
$T$ he role of particles in the fate and speciation of trace metals in macrotidal estuaries was studied using a surface complexation model (MOCO). Cadmium was selected as the target metal contaminant due to its reactivity in estuaries: cadmium behavior is mainly controlled by heterogeneous processes (sorption/desorption) related to salinity and suspended matter gradients. Various scenarios of suspended matter distribution according to salinity were simulated. The impact of surface properties (specific surface area, density of surface sites, acidity instrinsic properties and complexation constant) was evaluated using data collected on particles from the Gironde, Loire and Seine estuaries.
\end{abstract}

\section{I INTRODUCTION}

En milieu aquatique, les contaminants peuvent être présents sous forme dissoute, colloïdale et particulaire. La distinction entre ces trois formes est fixée de façon «opérationnelle » (par filtration, ultrafiltration) en fonction de leur taille. Au sein de ces catégories "physiques", les contaminants peuvent être sous différentes espèces «chimiques » en fonction de la nature et du type d'association. C'est la spéciation d'un contaminant qui sera responsable de sa biodisponibilité et de sa toxicité.

Différents facteurs physico-chimiques et biologiques contrôlent la spéciation et le passage de l'une à l'autre des différentes formes : le $\mathrm{pH}$; le potentiel redox ; la salinité ; la concentration dans l'eau de divers agents complexants; les caractéristiques sédimentologiques et la nature géochimique des particules.

L'importance des particules sur la dynamique des contaminants est d'autant plus importante dans les zones où les concentrations en matières en suspension (MES) sont élevées. A ce titre, les estuaires macrotidaux, caractérisés par de très importants gradients de MES et l'existence de zones d'accumulation des MES (bouchon vaseux), sont des systèmes où les particules pourront avoir un rôle majeur sur le devenir des contaminants. Dans ces systèmes, les concentrations en MES sont très variables, aussi bien d'un estuaire à un autre que au sein d'un même estuaire en fonction du débit fluvial et des conditions de marée. L'importance des MES sur les cycles biogéochimiques en milieu estuarien a été soulignée par Turner et Millward [2002], ainsi que le rôle de la salinité et des particules sur la distribution dissous particulaire des métaux traces [Turner, 1996].

Les concentrations en contaminants dans les particules estuariennes ne seront pas seulement le résultat des apports fluviaux, elles peuvent être fortement modifiées et régulées par des réactions à la surface des particules (sorption/désorp- tion) qui auront un rôle important sur la spéciation contaminants.

Le comportement des contaminants au cours du transit estuarien peut être classé de façon schématique: conservatif (comportement d'un élément qui va «passer à travers» un estuaire sans changer de phase) ou non-conservatif (s'il existe un processus qui change la distribution d'un contaminant).

Le cadmium est un très bon exemple de ce type de comportement. Cet élément est l'un des contaminants métalliques les plus réactifs en milieu estuarien. Il se caractérise, si l'on trace l'évolution longitudinale des concentrations dissoutes en fonction de la salinité, par une "courbe en cloche " [Boyle et al., 1982 ; Elbaz-Poulichet et al., 1987 ; Boutier et al., 1989 ; 1993 ; Chiffoleau et al., 1994 ; Kraepiel et al., 1997 ; Thouvenin et al., 1997 ; Chiffoleau et al., 2001a; 2001b].

La plupart des études menées sur le terrain ou en laboratoire [Comans and Van Dijk, 1988 ; Turner, 1996] indiquent que la désorption $\mathrm{du} \mathrm{Cd}$ du fait de la formation de chlorocomplexes explique la plus grande partie des observations. De plus, ce maximum de $\mathrm{Cd}$ dissous est d'autant plus marqué dans les estuaires macrotidaux où le temps de résidence des particules est élevé et où les concentrations en MES sont très importantes

L'ensemble de ces caractéristiques justifie le choix des estuaires macrotidaux et du cadmium pour «explorer» le rôle des propriétés des particules sur le devenir et la spéciation des contaminants métalliques.

L'objectif de la présentation est de mettre en évidence le rôle des particules, à travers leurs propriétés de surface (surface spécifique, nature et densité des sites de surface, propriétés acido-basiques, capacités de complexation) déterminées sur des particules issues de trois estuaires majeurs (la Seine, la Loire et la Gironde), sur le comportement et la spéciation de contaminants métalliques « réactifs ». 
Cette importance a été évaluée grâce à un modèle de complexation de surface (MOCO) qui est utilisé pour reproduire la spéciation de $\mathrm{Cd}$ au cours du transit estuarien en utilisant, dans différents scénarios, les propriétés de surface déterminées sur des particules prélevées dans les trois estuaires

\section{CARACTÉRISTIQUES GÉOCHIMIQUES ET PROPRIÉTÉS DE SURFACE DES PARTICULES}

La nature géochimique d'échantillons de particules prélevés dans les trois estuaires a été déterminée conjointement à l'évaluation de paramètres représentatifs de leurs propriétés de surface. La plupart des données, issues des travaux de Dange [2002], sont présentées dans le tableau 1.

Les concentrations des constituants majeurs indiquent que les particules de la Seine s'individualisent par les plus fortes teneurs en carbonates et carbone organique particulaire (COP). Celles de la Loire et de la Gironde ont des caractéristiques assez proches, sauf en ce qui concerne le COP et Mn.

Les valeurs des écarts type indiquent que le COP est la fraction la plus variable des particules, quelque soit l'estuaire. Les particules girondines sont les plus homogènes, ce qui pourrait être le résultat du fait que les échantillons ont été exclusivement prélevés dans la crème de vase, zone d'homogénéisation des particules les plus fines de l'estuaire.

Certaines propriétés de surface des particules naturelles, fortement liées à leurs caractéristiques minéralogiques, permettent de rendre compte de leur réactivité en milieu aquatique. La plupart de ces propriétés n'étant pas mesurables directement, leur estimation nécessite la mise en œuvre de différentes approches expérimentales. Ces paramètres, dont certains seront utilisés pour les simulations, sont présentés dans le tableau 1. Les techniques qui ont été mises en œuvre pour évaluer ces paramètres, ainsi que leurs limites sont décrites dans Gonzalez et al. [2001a ; 2001b] et Dange [2002].

Les particules de la Seine se caractérisent par la surface spécifique moyenne la plus faible, du fait en grande partie de leur granulométrie plus élevée [Dange, 2002] comme l'indiquent aussi les concentrations en $\mathrm{Al}, \mathrm{Fe}, \mathrm{Mn}$ qui sont les plus faibles et les fortes teneurs en carbonates.

La capacité d'échange cationique dépend de la composition géochimique des particules, notamment de la nature des minéraux argileux présents. Ce sont les échantillons girondins qui présentent les valeurs les plus élevées (Tab. 1 ), en accord avec la nature très argileuse de ces particules [Dange, 2002].

Ce sont les particules de la Seine qui présentent la densité de sites de surface la plus élevée. Les particules de la Loire, par rapport à celles de la Gironde, ont environ deux fois plus de sites de surface par mètre carré, ce qui malgré des caractéristiques très proches des particules de ces deux estuaires pourrait être le reflet des concentrations en Mn et COP plus élevées.

Dans le cas de la Seine, les résultats expérimentaux démontrent que les particules ne fixent pratiquement pas de protons. Les sites de surface seraient majoritairement non amphotères (Tab. 1), de type carboxylique et/ou phénoliques, caractéristiques de la matière organique (notamment de substances humiques). Au contraire, les matières en suspension de la Loire et de la Gironde fixent une quantité notable de protons.

n : nombre d'échantillons, SP : surface spécifique des particules, CEC : capacité d'échange cationique, SOHtot : concentration totale en sites actifs de surface, Ka1 et Ka2 : constantes d'acidité des sites de surface, $\mathbf{K m}$ : constante de complexation intrinsèque des sites de surface vis à vis de Cd. Ces paramètres sont associés aux équilibres suivants :

Pour les estuaires de la Gironde et de la Loire (un seul type «moyen » de sites de surface amphotères)

$$
\begin{gathered}
-\mathrm{SOH}_{+}{ }^{2} \Leftrightarrow-\mathrm{SOH}+\mathrm{H}^{+} \mathrm{Kal} \\
-\mathrm{SOH} \Leftrightarrow-\mathrm{SO}^{-}+\mathrm{Ka}^{2}
\end{gathered}
$$

Tableau 3. Caractéristiques géochimiques et propriétés de surface des particules : valeurs moyennes et écarts type (ET).

\begin{tabular}{|l|c|c|c|c|c|c|c|c|c|}
\hline \multirow{2}{*}{} & \multicolumn{3}{|c|}{ Seine } & \multicolumn{3}{c|}{ Loire } & \multicolumn{3}{c|}{ Gironde } \\
\cline { 2 - 12 } & Moyenne & ET (\%) & $\mathbf{n}$ & Moyenne & ET (\%) & N & Moyenne & ET (\%) & n \\
\hline CaCO3 \% & 26.8 & 13 & 24 & 7.9 & 11 & 5 & 8 & 3 & 11 \\
\hline Al \% & 3.4 & 32 & 45 & 7.7 & 28 & 25 & 8.9 & 20 & 30 \\
\hline Fe \% & 2.1 & 26 & 45 & 4.0 & 23 & 25 & 4.4 & 12 & 30 \\
\hline Mn $\mu \mathrm{g} / \mathrm{g}$ & 479 & 27 & 45 & 1056 & 29 & 25 & 865 & 19 & 30 \\
\hline COP \% & 5.1 & 70 & 45 & 4.3 & 44 & 25 & 1.6 & 49 & 30 \\
\hline SP ${ }^{2} / \mathrm{g}$ & 6.0 & 32 & 21 & 22.8 & 20 & 6 & 37.2 & 17 & 11 \\
\hline CEC mol/g & $1.69^{\mathrm{E}}-04$ & 30 & 24 & $2.37^{\mathrm{E}}-04$ & 39 & 6 & $3.47^{\mathrm{E}}-04$ & 26 & 11 \\
\hline$[-\mathbf{S O H t o t}] \mathrm{mol} / \mathrm{m}^{2}$ & $2.98^{\mathrm{E}}-05$ & 70 & 9 & $7.94^{\mathrm{E}}-06$ & 13 & 6 & $3.47^{\mathrm{E}}-06$ & 26 & 8 \\
\hline pKa1 & & & & 5.5 & 10 & 6 & 4.96 & 9 & 8 \\
\hline pKa2 & 4.59 & 13 & 10 & 6.72 & 8 & 6 & 6.42 & 7 & 8 \\
\hline Log Km & 0.48 & 52 & 7 & -1.51 & 45 & 4 & -1.21 & 22 & 5 \\
\hline
\end{tabular}


Pour l'estuaire de la Seine (un seul type «moyen » de sites de surface non amphotères)

$$
-\mathrm{SOH} \Leftrightarrow-\mathrm{SO}^{-}+\mathrm{H}^{+} \mathrm{Ka} 2
$$

Réaction de complexation de surface, sur des sites de type «moyens »

$$
-\mathrm{SOH}+\mathrm{Cd}^{2+} \Leftrightarrow-\mathrm{SOCd}^{+}+\mathrm{H}^{+} \mathrm{Km}
$$

Les propriétés acido-basiques de ces particules seront modélisées en considérant deux types de sites (Tab. 1): des sites de surface amphotères (hydroxyles) représentatifs des sites associés aux oxy-hydroxydes de Fe et Mn, ainsi qu'à la phase argileuse (fractions représentatives d'une partie importante des particules de la Loire et de la Gironde); et des sites non amphotères associés à la fraction organique qui contrôlent une grande partie du comportement acido-basique des particules de la Seine.

Les particules de la Seine ne présentent pas une teneur en COP très différente de celle des particules de la Loire. Mais l'importance de la fraction argileuse de ces dernières serait responsable de cette différence notable de comportement acido-basique.

Les données obtenues montrent que les propriétés acidobasiques des particules de la Loire et de la Gironde sont très proches, comme l'indiquent les constantes d'acidité (Tab. 1).

La valeur de Ka2 évaluée pour les échantillons de la Seine est représentative de sites carboxyliques et/ou phénoliques, elle confirme le rôle dominant de la matière organique sur la réactivité de ces particules.

L'ajustement des données issues des expériences de sorption de ${ }^{109} \mathrm{Cd}$ (Tab. 1) a permis d'évaluer la constante de complexation intrinsèque des sites de surface vis-à-vis de $\mathrm{Cd}$ $(\mathrm{Km})$. Pour les particules de la Loire et de la Gironde, les constantes sont proches, en accord avec la plupart des propriétés présentées précédemment. Les particules de la Seine présentent les valeurs moyennes les plus élevées.

\section{III — PRÉSENTATION DU MODÈLE UTILISÉ ET CONDITIONS DE SIMULATION}

Un modèle de complexation de surface (MOCO) est utilisé pour mettre en évidence le rôle des particules, à travers leurs propriétés de surface, sur la spéciation de $\mathrm{Cd}$ en milieu estuarien macrotidal. Ce type de modèle traite l'adsorption de $\mathrm{Cd}$ sur les particules comme une formation de complexes avec des groupes fonctionnels de surface [Stumm et al. 1980 ; Davis et Kent 1990 ; Dzombak and Morel 1987 ; 1990 ; Gonzalez et al., 2001a]. Les espèces dissoutes et particulaires de $\mathrm{Cd}$ sont calculées à partir de la résolution des équations d'équilibre faisant intervenir simultanément les différents ligands dissous et les particules. Les ligands dissous pris en compte sont: les chlorures, les hydroxydes et les sulfates. Les constantes de complexation utilisées sont issues de Comans and Van Dijk [1988] et Dzombak and Morel [1990].

Ce modèle a déjà été utilise pour simuler le comportement de $\mathrm{Cd}$ dans les trois estuaires choisis [Gonzalez et al., 2001a; 2001b]. Il a aussi utilisé pour modéliser le devenir d'autres cations métalliques comme $\mathrm{Co}$, Cs et $\mathrm{Hg}$ [Dange, 2002 ; Laurier et al., 2003].

Une description plus complète du modèle, l'obtention des paramètres utilisées et sa validation pour les différents estuaires abordés a été présentée par ailleurs [Gonzalez et al., 1999 ; 2001a ; 2001b ; Dange, 2002].

Les différentes distributions des concentrations en MES qui ont été mesurées dans les trois estuaires peuvent être schématisées en trois scénarios: une migration du maximum de turbidité vers les salinités les plus élevées; la présence d'un deuxième maximum de turbidité moins important et plus «étalé »; des pics de MES très élevés au sein de l'estuaire. Ce sont ces scénarios qui sont utilisés pour les simulations réalisées par la suite.

Toutes les simulations sont réalisées en utilisant les valeurs moyennes des paramètres de sorption (SA, [-SOHtot], Ka1, $\mathrm{Ka} 2, \mathrm{Km}$ ). Pour tester la variabilité naturelle de ces paramètres au sein d'un même estuaire, les simulations sont aussi réalisées en tenant compte de l'écart type autour des valeurs moyennes (Tab. 1).

Le modèle calcule, pour une concentration de $\mathrm{Cd}$ total donnée, les concentrations des différentes espèces (dissoutes et particulaires) à l'équilibre. Nous avons choisi une concentration en $\mathrm{Cd}$ total de $10^{-9} \mathrm{M}$ qui est représentative de ce que l'on peut mesurer dans les trois estuaires pris comme exemples.

Pour plus de clarté, les différentes espèces de $\mathrm{Cd}$ dissous (Cd libre, Cd complexé avec les chlorures, les sulfates ou les hydroxydes) ne sont pas présentées. Les résultats sont exprimés sous forme de $\mathrm{Cd}$ «dissous total» (somme de toutes les espèces dissoutes) et de $\mathrm{Cd}$ «particulaire ». Le $\mathrm{Cd}$ particulaire calculé par le modèle correspond à la fraction " adsorbée ».

\section{IV - PRINCIPAUX RESULATS}

\section{IV.1 CAPACITÉ GLOBALE DE SORPTION (GSC)}

Les capacités de sorption des particules sont fonction de: la capacité d'échange de protons des sites de surface évaluée grâce aux constantes d'acidité Ka1 et $\mathrm{Ka} 2$; la concentration totale en sites de surface et la surface spécifique des particules; la constante de complexation des sites de surface vis-à-vis de $\mathrm{Cd}$.

Afin de pouvoir comparer les capacités de sorption des particules issues des trois estuaires, et pouvoir interpréter les résultats obtenus, nous avons défini un paramètre représentatif de ces capacités : "la capacité globale de sorption» (CGS). Ce paramètre est calculé, en fonction de la salinité, à partir de la concentration en sites complexants $(-\mathrm{SOH}$, Tab. 1, eq. (4)) pour un $\mathrm{pH}$ de 7.8 (correspondant au $\mathrm{pH}$ des différentes simulations) et une concentration en MES de $100 \mathrm{mg} \mathrm{l}^{-1}$. Les calculs sont faits à partir des paramètres déterminés pour chaque type de particule: surface spécifique, densité des sites de surface, Ka1 et Ka2. La concentration en sites complexants ainsi obtenue est multipliée par la constante de complexation intrinsèque de Cd vis-à-vis des sites de surface $(\mathrm{Km})$. 
Seuls les résultats calculés avec les paramètres moyens sont présentés (figure 1). Ce sont les particules de la Loire qui ont les concentrations en sites complexants les plus élevées. Les particules de la Seine se caractérisent par les concentrations les plus faibles du fait du caractère non-amphotère des sites de surface (Tab. 1, eq. (3)).

Les valeurs de CGS pour Cd illustrent l'importance de la constante de complexation $(\mathrm{Km})$. La valeur importante de $\mathrm{Km}$ qui caractérise les particules de la Seine (Tab. 1), fait que malgré une très faible concentration en sites complexants, les CGS sont comparables aux particules des deux autres estuaires (Fig. 1).

Il existe plus d'un ordre de grandeur de différence entre les réactivités globales calculées à partir des paramètres de sorption moyens et celles calculées avec les paramètres minimum ou maximum (résultats non présentés ici). Avec les paramètres moyens et maxima, ce sont les particules de la Loire qui ont la réactivité globale la plus élevée, tandis qu'avec les paramètres minimums, ce sont les particules de la Gironde. Les particules de la Seine ont une réactivité très proche de celle des particules de la Gironde dans le cas des paramètres moyens et proche des particules de la Loire dans le cas des paramètres maximum. Il est à noter que les particules de la Seine, avec les paramètres moyens et maximum, se caractérisent par la plus forte réactivité à salinité nulle. Leur réactivité diminue très fortement dés que la salinité augmente, elle devient du même ordre que celle des autres particules vers salinité 1 .

Ce phénomène est expliqué par le fait que les sites de surface des particules de la Seine sont de type non amphotère (Tab. 1, eq. (3)). Une augmentation de la salinité entraîne une augmentation des constantes d'équilibre conditionnelles (Ka1, Ka2) du fait de la diminution des coefficients d'activité (leur diminution est la plus importante entre une force ionique de 0 et de quelques centièmes).

L'évaluation de la réactivité globale des particules met en évidence que les caractéristiques mesurées habituellement pour évaluer les capacités de sorption des particules vis-à- vis des contaminants (POC, Al, Fe, Mn, SA...) ne donnent que peu de renseignements dans ce domaine. Il faut accéder à des paramètres plus explicites (nature et densité des sites de surface, propriétés acido-basiques, constantes de complexation), ainsi qu'à leur variabilité naturelle, pour pouvoir évaluer dans différentes conditions physico-chimiques la réactivité des particules vis-à-vis d'un élément donné.

\section{IV.2 SIMULATION DE LA MIGRATION DU MAXIMUM DE TURBIDITÉ}

La migration de la zone de turbidité maximale, en fonction des conditions de débit et de marée, dans une gamme de salinité relativement faible est une situation fréquemment rencontrée dans les estuaires macrotidaux.

Avec les propriétés moyennes, les résultats obtenus sont très proches quelque soit le type de particule (Fig. 2[A]), notamment pour la Seine et la Loire. Les différences sont importantes à salinité nulle quand les teneurs en MES sont faibles $(25 \mathrm{mg} / \mathrm{l})$. Comme l'indique l'évaluation de la réactivité globale des particules, les particules de la Seine présentent des propriétés de sorption nettement plus élevées à salinité nulle

\section{IV.3 EFFET DE LA PRÉSENCE D'UN SECOND PIC DE TURBIDITÉ}

Ce scénario va permettre d'évaluer l'effet d'un deuxième maximum de turbidité dans des eaux salinité plus élevée, cette situation résultat de facteurs hydrodynamiques peut être rencontrée fréquemment dans les estuaires macrotidaux, c'est notamment le cas des estuaires de la Loire et de la Gironde.

Dans le cas des propriétés moyennes (fig. 2[B]), les différences entre la nature des particules ne sont notables qu'à salinité 0 . Le pic de turbidité le plus important est bien marqué par un minimum du pourcentage en $\mathrm{Cd}$ dissous. L'effet du deuxième maximum de turbidité se traduit par une légère inflexion de la courbe de $\% \mathrm{Cd}$.
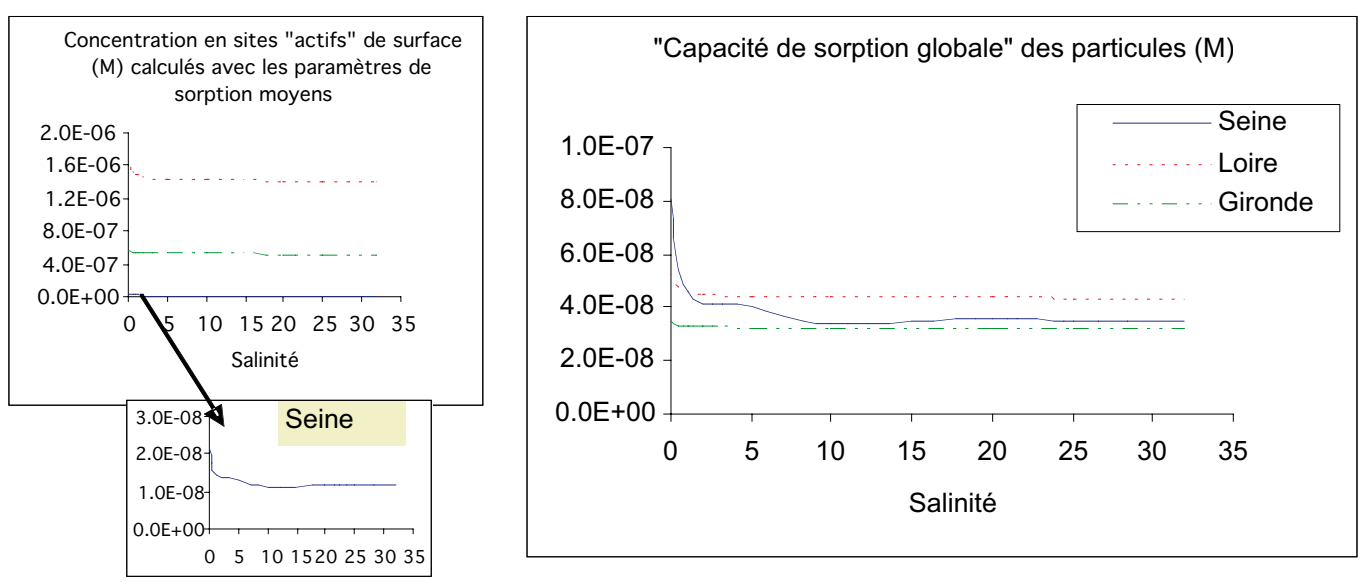

Figure 1 : Concentration en sites actifs de surface et capacité de sorption globale des particules. Les calculs ont été réalisés en utilisant les propriétés moyennes de sorption des particules. 


\section{IV.4 SIMULATION DE LA PRÉSENCE DE DIFFÉRENTS PICS DE TURBIDITÉ}

La présence de pics importants de turbidité, dans n'importe quelle gamme de salinité, est une situation que l'on
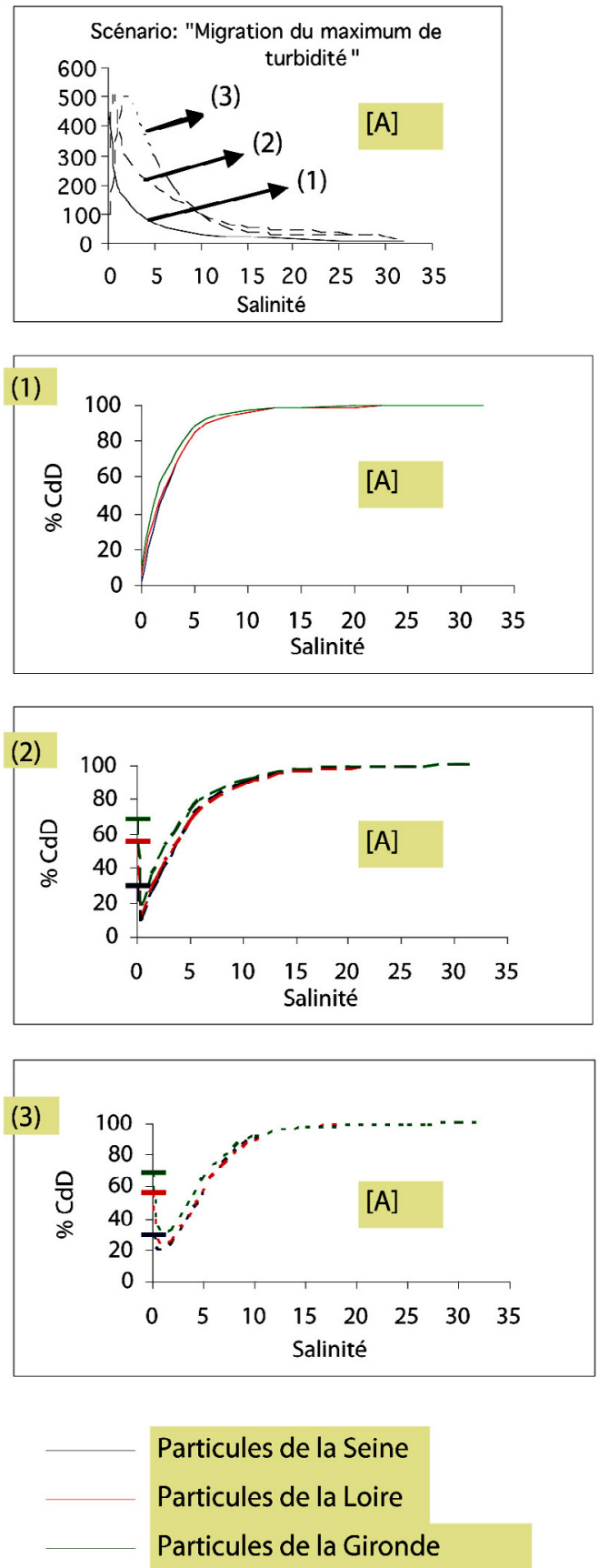

rencontre souvent dans les cas où des zones de dépôt de vases sont érodées.

L'effet des pics de turbidité est important, même dans les eaux de salinité élevée, avec les propriétés de sorption moyennes (fig. 2[C]).
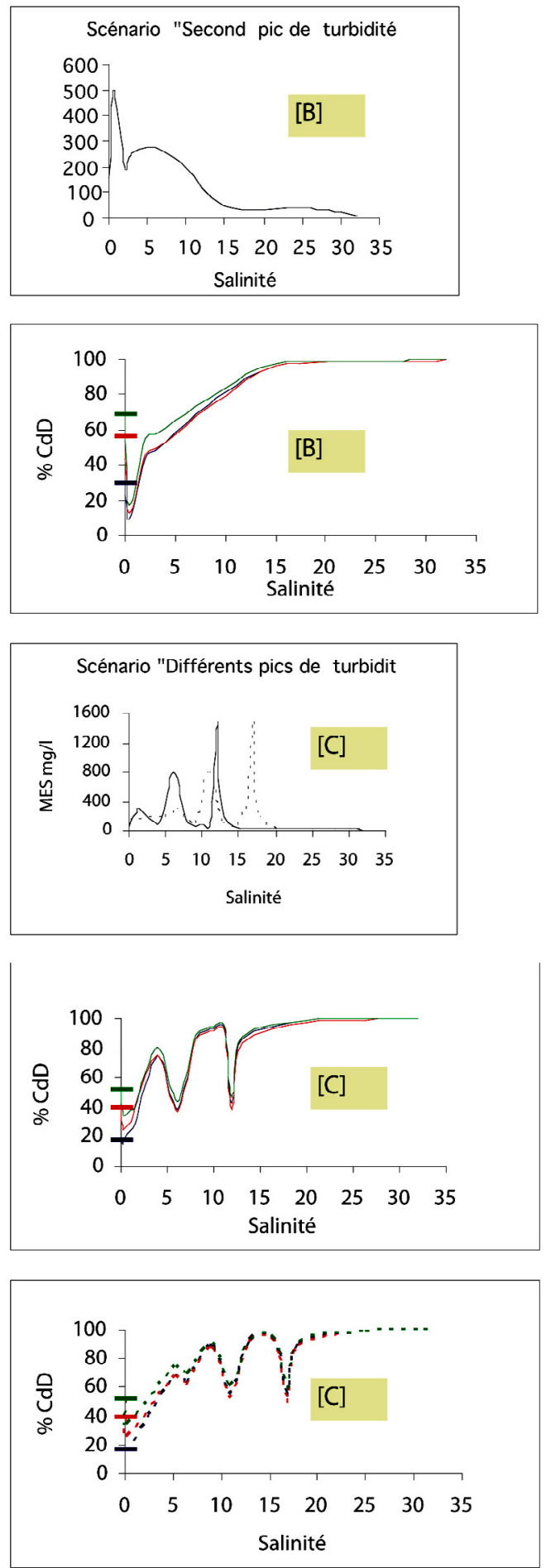

Figure 2 : Pourcentage de Cd dissous (\%CdD). Simulations réalisées avec les propriétés de sorption moyennes :

[A] Scénario « Migration du maximum de turbidité »; [B] Scénario « Second pic de turbidité »;

[C] Scénario « Différents pics de turbidité ». 


\section{V $\square$ CONCLUSIONS}

$\mathrm{Du}$ fait de sa réactivité, le cadmium a été choisi pour explorer le rôle des particules, à travers leurs propriétés de surface sur le devenir et la spéciation des contaminants dans les estuaires macrotidaux. Les simulations du comportement de cet élément, très sensible aux échanges dissous/particules, en utilisant les propriétés de sorption représentatives de particules issues de différents estuaires, pour montre que :

- Même quand les capacités de sorption sont faibles et que le comportement de $\mathrm{Cd}$ est pratiquement conservatif dans l'estuaire, si les concentrations en MES sont importantes et que la salinité est proche de zéro, les différences en fonction de l'origine des particules peut être très importante notamment dans le cas où il y a des écarts importants (de l'ordre d'un facteur 7) entre la réactivité globale des particules.

- Dans le cas des propriétés moyennes (représentatives de l'ensemble des mesures réalisées sur différents échantillons), $\mathrm{du}$ fait qu'il n'y a relativement peu de variations entre la réactivité globale des particules, la simulation de la partition dissous/particules donne des résultats comparables quelque soit l'origine des particules. L'ensemble des scénarios simulés met en évidence l'importance des particules, quelque soit la salinité.

- Quand les propriétés de sorption sont élevées, les différences entre les particules auront beaucoup d'importance sur le comportement de $\mathrm{Cd}$, même pour des salinités élevées.

L'évaluation des capacités globales de sorption des particules (CGS) met en évidence que les paramètres utilisés habituellement pour évaluer l'affinité des contaminants pour les particules (POC, $\mathrm{Al}, \mathrm{Fe}, \mathrm{Mn}$, surface spécifique, etc.) ne donnent en fait que relativement peu d'indications et ne permettent pas de comparer des particules en terme de capacités de sorption.

L'influence des propriétés de surface des particules est maximale quand la salinité tend vers 0 et que la concentration en MES est élevée. Quand la salinité augmente, du fait de l'importance des chlorures sur la spéciation de $\mathrm{Cd}$, le rôle de la phase particulaire est lissé. Cela implique que pour des éléments de type $\mathrm{Cd}$, dont le comportement est contrôlé par la concurrence entre la phase particulaire et un ligand important associé à la phase dissoute (par exemple l'ion chlorure dans le cas de $\mathrm{Cd}$ ), les propriétés de sorption des particules auront un rôle majeur sur la spéciation de ces éléments. Ainsi dans les estuaires macrotidaux, les variations spatio-temporelles, par rapport au gradient de salinité, de la quantité en MES et de leurs propriétés contribueront à la partition dissous/particulaire et aux flux de ces éléments à l'interface océan continent.

Dans une optique de modélisation, les résultats obtenus indiquent qu'il est possible de simuler (et de prévoir), en évaluant expérimentalement un certain nombre de paramètres, le comportement de contaminants comme le cadmium dans des milieux complexes. Ces paramètres caractérisent, les processus (constante de complexation de surface, constantes d'acidité des sites de surface) et les particules (densité de sites de surface). Leur valeur doit être déterminée avec suf- fisamment de précision et leur variabilité au sein du milieu doit être connue.

Il est à noter que les tests présentés ne prennent pas en compte l'aspect dynamique des processus, notamment l'évolution temporelle des gradients de turbidité et de salinité qui est très importante dans les estuaires macrotidaux. Ne sont considérés dans cette étude que le rôle des particules dans les processus proches de l'équilibre. Des processus «lents », dont les particules sont le moteur, peuvent aussi avoir une influence importante contrôler la dynamique et la spéciation des métaux traces en milieu estuarien: désorption, processus liés à la minéralisation de certaines phases minérales (oxydes de Fe et Mn, matière organique), notamment dans les zones de dépôt de matériel fin et dans les zones de turbidité maximale. Néanmoins les résultats obtenus montrent que les propriétés de surface des particules ont une grande importance, leur évaluation permet de comprendre et simuler une grande partie du devenir des contaminants «non conservatifs » en milieu estuarien.

\section{REFERENCES}

[1] Boyle E.A., Edmond J. M. \& Sholkovitz E.R. (1982). — The mechanism of iron removal in estuaries. Geochim. Cosmochim. Acta, 41 : 1313-1324.

[2] Boutier B., Jounnneau J.M., Chiffoleau J.F., Latouche C. \& Phillips I. (1989). — La contamination de la Gironde par le cadmium. Rapports Scientifiques et Techniques de l'IFREMER, $14: 105 \mathrm{p}$.

[3] Boutier B., Chiffoleau J.F., Auger D. \& Truquet I. (1993). - Influence of the Loire River on dissolved Lead and Cadmium concentrations in coastal waters of Brittany. Est. Cstl. Mar. Sci., 36 : 133-145.

[4] Chiffoleau J.F., Cossa D., Auger D. \& Truquet I. (1994). - Trace metal distribution, partition and fluxes in the Seine estuary (France) in low discharge regime. Mar. Chem., 47 : 145-158.

[5] Chiffoleau J.F., Auger D., Chartier E., Michel P., Truquet I., Ficht A., Gonzalez J.L. \& Romana L.A. (2001)a. Spatiotemporal changes in cadmium contamination in the Seine Estuary (France). Estuaries, 24, 6B, 1029-1040.

[6] Chiffoleau J.-F., Claisse D., Cossa D., Ficht A., Gonzalez J.-L., Guyot T., Michel P., Miramand P., Oger C. \& Petit F. (2001)b. - La contamination métallique. Programme scientifique "Seine Aval ». Fascicule no 8 . Editions IFREMER. Plouzané (France). 39 p.

[7] Comans R.N.J. \& VAN DiJk C.P.J. (1988). — Role of complexation processes in cadmium mobilization during estuarine mixing. Nature, 336 : 151-154.

[8] Dange C. (2002). - Etude du comportement des éléments traces en milieu estuarien: approche expérimentale et modélisation. Thèse de doctorat de l'Université de Reims ChampagneArdenne, $467 \mathrm{p}$.

[9] Davis J.A. \& Kent D.B. (1990). - Surface complexation modelling in aqueous geochemistry. In M. F. Hochella \& A. F. White (eds.), Mineral-water interface geochemistry, Reviews in Mineralogy, 23 : 177-260. 
[10] Dzombak D.A. \& Morel F.M.M. (1987). - Adsorption of inorganic pollutants in aquatic systems. J. Hydraul. Eng., 113 : 430-475.

[11] Dzombak D.A. \& Morel F.M.M. (1990). - Surface complexation modeling: Hydrous ferric oxide. John Wiley, New York, $393 \mathrm{p}$.

[12] Elbaz-Poulichet F., Huang W.W., Martin J.M. \& Zhu J.X. (1987). - Dissolved cadmium behaviour in some selected french and chinese estuaries. Mar. Chem. 22 : 125-136.

[13] Gonzalez J.L., Thouvenin B., Chiffoleau J.F. \& Miramand P. (1999). — Le cadmium : comportement d'un contaminant métallique en estuaire. Programme Scientifique "Seine Aval ». Fascicule $\mathrm{n}^{\mathrm{0}} \mathbf{1 0}$. Editions IFREMER. Plouzané (France). 31 p.

[14] Gonzalez J.L., Thouvenin B., Dange C., Fiandrino A. \& Chiffoleau J.F. (2001)a. - Modeling of Cd speciation and dynamics in the Seine estuary (France). Estuaries, 24, 6B : 1041-1055.

[15] Gonzalez J.L., Dange C. \& Thouvenin B. (2001)b. Spéciation des contaminants métalliques en milieu estuarien : Intérêt de la modélisation et application au cadmium. Hydroécologie Appliquée, 13, vol. 1 : 37-55.
[16] Kraepiel A.M.L., Chiffoleau J.F., Martin J.M. \& Morel F.M.M. (1997). - Geochemistry of trace metals in the Gironde estuary. Geochimica Cosmochimica Acta, 61 : 1421-1436.

[17] Laurier F.J.G., Cossa D., Gonzalez J.L., Breviere E. \& SARAZIN G. (2003). - Mercury transformations and exchanges in a high turbidity estuary: the role of organic matter and amorphous oxyhydroxides. Geochimica Cosmochimica Acta, 67, $18: 3329-3345$.

[18] Stumm W., Kummer R. \& Sigg L. (1980). — A ligand exchange model for the adsorption of inorganic and organic ligands at hydrous oxide interfaces. Croat. Chem. Acta, 53 : 291-312.

[19] Thouvenin B., Gonzalez J.L. \& Boutier B. (1997). Modelling of pollutants behaviour in estuaries. Mar. Chem., 58, $\mathrm{n}^{\circ} 1-2: 147-161$.

[20] Turner A. \& Millward G.E. (2002). - Suspended particles : Their role in estuarine biogeochemical cycles. Estuarine, Coastal and Shelf Science, 55 : 857-883.

[21] TuRner A. (1996). - Trace-metal partitioning in estuaries : importance of salinity and particle concentration. Mar. Chem., 54, $\mathrm{n}^{\circ} 1-2:$ 27-39. 